$\operatorname{Nr} 60$

George A. Anastassiou

\title{
APPROXIMATION BY MAX-PRODUCT OPERATORS
}

\begin{abstract}
Here we study the approximation of functions by a great variety of Max-Product operators under differentiability. These are positive sublinear operators. Our study is based on our general results about positive sublinear operators. We produce Jackson type inequalities under initial conditions. So our approach is quantitative by producing inequalities with their right hand sides involving the modulus of continuity of a high order derivative of the function under approximation. We improve known related results which do not use smoothness of functions..

KEY WORDS: positive sublinear operators, Max-product operators, modulus of continuity.
\end{abstract}

AMS Mathematics Subject Classification: 41A17, 41A25, 41A36.

\section{Introduction}

The main motivation here comes from the monograph by B. Bede, L. Coroianu and S. Gal [3], 2016.

We mention the interpolation Hermite-Fejer polynomials on Chebyshev knots of the first kind (see [3], p. 4): Let $f:[-1,1] \rightarrow \mathbb{R}$ and based on the knots $x_{N, k}=\cos \left(\frac{(2(N-k)+1)}{2(N+1)} \pi\right) \in(-1,1), k \in\{0, \ldots, N\},-1<x_{N, 0}<$ $x_{N, 1}<\ldots<x_{N, N}<1$, which are the roots of the first kind Chebyshev polynomial $T_{N+1}(x)=\cos ((N+1) \arccos x)$, we define (see Fejér [4])

$$
H_{2 N+1}(f)(x)=\sum_{k=0}^{N} h_{N, k}(x) f\left(x_{N, k}\right),
$$

where

$$
h_{N, k}(x)=\left(1-x \cdot x_{N, k}\right)\left(\frac{T_{N+1}(x)}{(N+1)\left(x-x_{N, k}\right)}\right)^{2}
$$

the fundamental interpolation polynomials. 
Denoting $A_{N+1}(f)=\left\|H_{2 N+1}-f\right\|_{\infty}$, Fejer [4] proved that $\lim _{N \rightarrow \infty} A_{N+1}(f)$ $=0$, for all $f \in C([-1,1])$.

Popoviciu ([6]) also proved that $A_{N+1}(f)=O\left(\omega_{1}\left(f, \frac{1}{\sqrt{N+1}}\right)\right)$, and Moldovan ([5]) improved it to

$$
A_{N+1}(f)=O\left(\omega_{1}\left(f, \frac{\ln (N+1)}{N+1}\right)\right) .
$$

Here $\omega_{1}(f, \delta)=\sup _{\substack{x, y \in[-1,1]: \\|x-y| \leq \delta}}|f(x)-f(y)|, \delta>0$, is the first modulus of continuity.

The Max-product interpolation Hermite-Fejér operators on Chebyshev knots of the first kind (see p. 12 of [3]) are defined by

$$
H_{2 N+1}^{(M)}(f)(x)=\frac{\bigvee_{k=0}^{N} h_{N, k}(x) f\left(x_{N, k}\right)}{\bigvee_{k=0}^{N} h_{N, k}(x)}, \forall N \in \mathbb{N},
$$

where $f:[-1,1] \rightarrow \mathbb{R}_{+}$is continuous.

By [3], p. 286 we get that

$$
\begin{aligned}
& \left|H_{2 N+1}^{(M)}(f)(x)-f(x)\right| \\
& \leq 14 \omega_{1}\left(f, \frac{1}{N+1}\right), \quad \forall N \in \mathbb{N} \text {, any } x \in[-1,1] .
\end{aligned}
$$

Call

$$
\begin{aligned}
E_{N}(x) & :=H_{2 N+1}^{(M)}(|\cdot-x|)(x) \\
& =\frac{\bigvee_{k=0}^{N} h_{N, k}(x)\left|x_{N, k}-x\right|}{\bigvee_{k=0}^{N} h_{N, k}(x)}, x \in[-1,1] .
\end{aligned}
$$

Then by [3], p. 287 we obtain that

$$
E_{N}(x) \leq \frac{2 \pi}{N+1}, \forall x \in[-1,1], \quad N \in \mathbb{N} .
$$

For $m \in \mathbb{N}$, we get

$$
\begin{aligned}
H_{2 N+1}^{(M)}\left(|\cdot-x|^{m}\right)(x) & =\frac{\bigvee_{k=0}^{N} h_{N, k}(x)\left|x_{N, k}-x\right|^{m}}{\bigvee_{k=0}^{N} h_{N, k}(x)} \\
& =\frac{\bigvee_{k=0}^{N} h_{N, k}(x)\left|x_{N, k}-x\right|\left|x_{N, k}-x\right|^{m-1}}{\bigvee_{k=0}^{N} h_{N, k}(x)} \\
& \leq 2^{m-1} \frac{\bigvee_{k=0}^{N} h_{N, k}(x)\left|x_{N, k}-x\right|}{\bigvee_{k=0}^{N} h_{N, k}(x)} \\
& \leq \frac{2^{m} \pi}{N+1} \quad \forall x \in[-1,1], \quad N \in \mathbb{N}
\end{aligned}
$$


Hence it holds

$$
H_{2 N+1}^{(M)}\left(|\cdot-x|^{m}\right)(x) \leq \frac{2^{m} \pi}{N+1}, \forall x \in[-1,1], m \in \mathbb{N}, \quad \forall N \in \mathbb{N} .
$$

Clearly it holds

$$
H_{2 N+1}^{(M)}\left(|\cdot-x|^{m}\right)(x)>0
$$

$\forall x \in[-1,1]: x \neq x_{N, k}, \forall N \in \mathbb{N}$, any $k \in\{0,1, \ldots, N\} ;$ any $m \in \mathbb{N}$.

Furthermore we have

$$
H_{2 N+1}^{(M)}(1)(x)=1, \quad \forall x \in[-1,1],
$$

and $H_{2 N+1}^{(M)}$ maps continuous functions to continuous functions over $[-1,1]$ and for any $x \in \mathbb{R}$ we have $\bigvee_{k=0}^{N} h_{N, k}(x)>0$.

We also have $h_{N, k}\left(x_{N, k}\right)=1$, and $h_{N, k}\left(x_{N, j}\right)=0$, if $k \neq j$, furthermore it holds $H_{2 N+1}^{(M)}(f)\left(x_{N, j}\right)=f\left(x_{N, j}\right)$, for all $j \in\{0, \ldots, N\}$, see [3], p. 282 .

In this work we will improve (4) by assuming differentiability of $f$. Similar improvements, using the differentiability of $f$, will be presented for Max-product Lagrange interpolation operators, Max-product truncated sampling operators and Max-product Neural network operators.

\section{Main results}

Let $I \subset \mathbb{R}$ be a bounded or unbounded interval, $n \in \mathbb{N}$, and

(10) $C B_{+}^{n}(I)=\left\{f: I \rightarrow \mathbb{R}_{+}: f^{(i)}\right.$ is continuous and bounded on $I$,

$$
\text { for both } i=0, n\} \text {. }
$$

We define for

(11) $f \in C B_{+}(I)=\left\{f: I \rightarrow \mathbb{R}_{+}: f\right.$ is continuous and bounded on $\left.I\right\}$,

the first modulus of continuity

$$
\omega_{1}(f, \delta)=\sup _{\substack{x, y \in I: \\|x-y| \leq \delta}}|f(x)-f(y)|
$$

where $0<\delta \leq$ diameter $(I)$.

Call $C_{+}(I)=\left\{f: I \rightarrow \mathbb{R}_{+}: f\right.$ is continuous on $\left.I\right\}$.

Let $L_{N}: C_{+}(I) \rightarrow C B_{+}(I), n, N \in \mathbb{N}$ be a sequence of operators satisfying the following properties (see also [3], p. 17): 
(i) (positive homogeneous)

$$
L_{N}(\alpha f)=\alpha L_{N}(f), \quad \forall \alpha \geq 0, \quad f \in C_{+}(I),
$$

(ii) (Monotonicity)

(14) if $f, g \in C_{+}(I)$ satisfy $f \leq g$, then $L_{N}(f) \leq L_{N}(g), \forall N \in \mathbb{N}$,

and

(iii) (Subadditivity)

$$
L_{N}(f+g) \leq L_{N}(f)+L_{N}(g), \quad \forall f, g \in C_{+}(I) .
$$

We call $L_{N}$ positive sublinear operators.

In particular we will study the restrictions $\left.L_{N}\right|_{C B_{+}^{n}(I)}: C B_{+}^{n}(I) \rightarrow$ $C B_{+}(I)$.

The operators $H_{2 N+1}^{(M)}$ are positive sublinear operators. From [1] we will be using the following result:

Theorem 1 ([1]). Let $\left(L_{N}\right)_{N \in \mathbb{N}}$ be a sequence of positive sublinear operators from $C_{+}(I)$ into $C B_{+}(I)$, and $f \in C B_{+}^{n}(I)$, where $n \in \mathbb{N}$ and $I \subset \mathbb{R}$ a bounded or unbounded interval. Assume $L_{N}(1)=1, \forall N \in \mathbb{N}$, and $f^{(i)}(x)=0, i=1, \ldots, n$, for a fixed $x \in I$, and $\delta>0$. Then

$$
\begin{aligned}
& \left|L_{N}(f)(x)-f(x)\right| \\
& \quad \leq \frac{\omega_{1}\left(f^{(n)}, \delta\right)}{n !}\left[L_{N}\left(|\cdot-x|^{n}\right)(x)+\frac{L_{N}\left(|\cdot-x|^{n+1}\right)(x)}{(n+1) \delta}\right],
\end{aligned}
$$

$\forall N \in \mathbb{N}$

We give

Theorem 2. Let $f \in C^{n}\left([-1,1], \mathbb{R}_{+}\right)$, with $f^{(i)}(x)=0, i=1, \ldots, n \in \mathbb{N}$, for some fixed $x \in[-1,1], N \in \mathbb{N}$. Then

$$
\begin{aligned}
& \left|H_{2 N+1}^{(M)}(f)(x)-f(x)\right| \\
& \quad \leq \frac{1}{n !} \omega_{1}\left(f^{(n)}, 2 \sqrt[n+1]{\frac{\pi}{N+1}}\right)\left[\frac{2^{n} \pi}{N+1}+\frac{2^{n}}{(n+1)}\left(\sqrt[n+1]{\frac{\pi}{N+1}}\right)^{n}\right] .
\end{aligned}
$$

When $x=x_{N, k}$, the left hand side of (17) is zero.

Proof. Here we are using (8) and (16), namely we have

$$
H_{2 N+1}^{(M)}\left(|\cdot-x|^{m}\right)(x) \leq \frac{2^{m} \pi}{N+1}, \quad \forall x \in[-1,1], \quad m \in \mathbb{N}, \quad \forall N \in \mathbb{N} .
$$


and

$$
\begin{aligned}
& \left|H_{2 N+1}^{(M)}(f)(x)-f(x)\right| \\
& \quad \leq \frac{\omega_{1}\left(f^{(n)}, \delta\right)}{n !}\left[\frac{2^{n} \pi}{N+1}+\frac{1}{(n+1) \delta}\left(\frac{2^{n+1} \pi}{N+1}\right)\right]
\end{aligned}
$$

$\delta>0$.

Then choose

$$
\delta:=2 \sqrt[n+1]{\frac{\pi}{N+1}}
$$

we get

$$
\delta^{n+1}=2^{n+1} \frac{\pi}{N+1},
$$

and

$$
\begin{aligned}
& \left|H_{2 N+1}^{(M)}(f)(x)-f(x)\right| \leq \frac{\omega_{1}\left(f^{(n)}, 2 \sqrt[n+1]{\frac{\pi}{N+1}}\right)}{n !}\left[\frac{2^{n} \pi}{N+1}+\frac{1}{(n+1)} \delta^{n}\right] \\
& \quad=\frac{1}{n !} \omega_{1}\left(f^{(n)}, 2 \sqrt[n+1]{\frac{\pi}{N+1}}\right)\left[\frac{2^{n} \pi}{N+1}+\frac{1}{(n+1)} 2^{n}\left(\sqrt[n+1]{\frac{\pi}{N+1}}\right)^{n}\right]
\end{aligned}
$$

proving the claim.

It follows the $n=1$ case.

Corollary 1. Let $f \in C^{1}\left([-1,1], \mathbb{R}_{+}\right)$, with $f^{\prime}(x)=0$, for some fixed $x \in[-1,1], N \in \mathbb{N}$. Then

$$
\begin{aligned}
& \left|H_{2 N+1}^{(M)}(f)(x)-f(x)\right| \\
& \quad \leq \omega_{1}\left(f^{\prime}, 2 \sqrt{\frac{\pi}{N+1}}\right)\left[\frac{2 \pi}{N+1}+\sqrt{\frac{\pi}{N+1}}\right] .
\end{aligned}
$$

From (17) and/or (23), as $N \rightarrow \infty$, we get that $H_{2 N+1}^{(M)}(f)(x) \rightarrow f(x)$.

Proof. By (17).

We make

Remark 1. We compare (23) to (4). We prove that (23) gives a sharper estimate and speed than (4). We observe that

$$
\frac{2 \sqrt{\pi}}{\sqrt{N+1}}\left(\frac{2 \pi}{N+1}+\frac{\sqrt{\pi}}{\sqrt{N+1}}\right) \leq \frac{14}{N+1}
$$




$$
\begin{gathered}
\Leftrightarrow \\
\frac{1}{\sqrt{N+1}} \leq \frac{7-\pi}{2 \pi \sqrt{\pi}},
\end{gathered}
$$

true for large enough $N \in \mathbb{N}$.

We also make

Remark 2. Here we compare (17) to (4). We prove that (17) gives a better estimate and speed than (4). We see that

$$
\begin{gathered}
\frac{2 \sqrt[n+1]{\pi}}{n ! \sqrt[n+1]{N+1}}\left[\frac{2^{n} \pi}{N+1}+\frac{2^{n}}{(n+1)}\left(\frac{\sqrt[n+1]{\pi}}{\sqrt[n+1]{N+1}}\right)^{n}\right] \leq \frac{14}{N+1}, \\
\frac{1}{\sqrt[n+1]{N+1}} \leq\left(7-\frac{2^{n} \pi}{(n+1) !}\right) \frac{n !}{2^{n} \pi \sqrt[n+1]{\pi}}
\end{gathered}
$$

true for large enough $N \in \mathbb{N}$.

About notice that $7-\frac{2^{n} \pi}{(n+1) !}>0$.

We continue with

Remark 3. Here we deal with Lagrange interpolation polynomials on Chebyshev knots of second kind plus the endpoints \pm 1 (see [3], p. 5). These polynomials are linear operators attached to $f:[-1,1] \rightarrow \mathbb{R}$ and to the knots $x_{N, k}=\cos \left(\left(\frac{N-k}{N-1}\right) \pi\right) \in[-1,1], k=1, \ldots, N, N \in \mathbb{N}$, which are the roots of $\omega_{N}(x)=\sin (N-1) t \sin t, x=\cos t$. Notice that $x_{N, 1}=-1$ and $x_{N, N}=1$. Their formula is given by ([3], p. 377)

$$
L_{N}(f)(x)=\sum_{k=1}^{N} l_{N, k}(x) f\left(x_{N, k}\right)
$$

where

$$
l_{N, k}(x)=\frac{(-1)^{k-1} \omega_{N}(x)}{\left(1+\delta_{k, 1}+\delta_{k, N}\right)(N-1)\left(x-x_{N, k}\right)},
$$

$N \geq 2, k=1, \ldots, N$, and $\omega_{N}(x)=\prod_{k=1}^{N}\left(x-x_{N, k}\right)$ and $\delta_{i, j}$ denotes the Kronecher's symbol, that is $\delta_{i, j}=1$, if $i=j$, and $\delta_{i, j}=0$, if $i \neq j$. Then (see [3], p. 5)

$$
\left\|L_{N}(f)-f\right\|_{\infty,[-1,1]} \leq c \omega_{1}\left(f, \frac{1}{N}\right) \ln N
$$


The Max-product Lagrange interpolation operators on Chebyshev knots of second kind, plus the endpoints \pm 1 , are defined by ([3], p. 12)

$$
L_{N}^{(M)}(f)(x)=\frac{\bigvee_{k=1}^{N} l_{N, k}(x) f\left(x_{N, k}\right)}{\bigvee_{k=1}^{N} l_{N, k}(x)}, x \in[-1,1],
$$

where $f:[-1,1] \rightarrow \mathbb{R}_{+}$is continuous.

First we see that $L_{N}^{(M)}(f)(x)$ is well defined and continuous for any $x \in[-1,1]$. Following [3], p. 289, because $\sum_{k=1}^{N} l_{N, k}(x)=1, \forall x \in \mathbb{R}$, for any $x$ there exists $k \in\{1, \ldots, N\}: l_{N, k}(x)>0$, hence $\bigvee_{k=1}^{N} l_{N, k}(x)>0$. We have that $l_{N, k}\left(x_{N, k}\right)=1$, and $l_{N, k}\left(x_{N, j}\right)=0$, if $k \neq j$. Furthermore it holds $L_{N}^{(M)}(f)\left(x_{N, j}\right)=f\left(x_{N, j}\right)$, all $j \in\{1, \ldots, N\}$, and $L_{N}^{(M)}(1)=1$.

Call $I_{N}^{+}(x)=\left\{k \in\{1, \ldots, N\} ; l_{N, k}(x)>0\right\}$, then $I_{N}^{+}(x) \neq \emptyset$.

So for $f \in C B_{+}([-1,1])$ we get

$$
L_{N}^{(M)}(f)(x)=\frac{\bigvee_{k \in I_{N}^{+}(x)} l_{N, k}(x) f\left(x_{N, k}\right)}{\bigvee_{k \in I_{N}^{+}(x)} l_{N, k}(x)} \geq 0 .
$$

By [3], p. 295, we have:

Let $f \in C\left([-1,1], \mathbb{R}_{+}\right), N \in \mathbb{N}, N \geq 3, N$ is odd, then

$$
\left|L_{N}^{(M)}(f)(x)-f(x)\right| \leq 4 \omega_{1}\left(f, \frac{1}{N-1}\right), \quad \forall x \in[-1,1] .
$$

Notice here that $\left|x_{N, k}-x\right| \leq 2, \forall x \in[-1,1]$.

By [3], p. 297, we get that

$$
\begin{aligned}
L_{N}^{(M)}(|\cdot-x|)(x) & =\frac{\bigvee_{k=1}^{N} l_{N, k}(x)\left|x_{N, k}-x\right|}{\bigvee_{k=1}^{N} l_{N, k}(x)} \\
& =\frac{\bigvee_{k \in I_{N}^{+}(x)} l_{N, k}(x)\left|x_{N, k}-x\right|}{\bigvee_{k \in I_{N}^{+}(x)} l_{N, k}(x)} \leq \frac{\pi^{2}}{6(N-1)},
\end{aligned}
$$

$N \geq 3, \forall x \in(-1,1), N$ is odd.

We get that $(m \in \mathbb{N})$

$$
L_{N}^{(M)}\left(|\cdot-x|^{m}\right)(x)=\frac{\bigvee_{k \in I_{N}^{+}(x)} l_{N, k}(x)\left|x_{N, k}-x\right|^{m}}{\bigvee_{k \in I_{N}^{+}(x)} l_{N, k}(x)} \leq \frac{2^{m-1} \pi^{2}}{6(N-1)},
$$

$N \geq 3$ odd, $\forall x \in(-1,1)$.

We present 
Theorem 3. Let $f \in C^{n}\left([-1,1], \mathbb{R}_{+}\right), n \in \mathbb{N}, x \in[-1,1], f^{(i)}(x)=0$, $i=1, \ldots, n$. Here $N \in \mathbb{N}, N \geq 3$ is odd. Then

$$
\begin{aligned}
(36) \mid & L_{N}^{(M)}(f)(x)-f(x) \mid \\
& \leq \frac{\omega_{1}\left(f^{(n)},\left(\frac{2^{n} \pi^{2}}{6(N-1)}\right)^{\frac{1}{n+1}}\right)}{n !}\left[\frac{2^{n-1} \pi^{2}}{6(N-1)}+\frac{1}{(n+1)}\left(\frac{2^{n} \pi^{2}}{6(N-1)}\right)^{\frac{n}{n+1}}\right] .
\end{aligned}
$$

Proof. When $x= \pm 1$, the left hand side of (36) is zero, hence (36) is trivially true. Let now $x \in(-1,1)$, by Theorem 1 and (16), (35), we obtain

$$
\begin{aligned}
& \left|L_{N}^{(M)}(f)(x)-f(x)\right| \\
& \quad \leq \frac{\omega_{1}\left(f^{(n)}, \delta\right)}{n !}\left[\frac{2^{n-1} \pi^{2}}{6(N-1)}+\frac{1}{(n+1) \delta} \frac{2^{n} \pi^{2}}{6(N-1)}\right]
\end{aligned}
$$

$\left(\right.$ setting $\delta:=\left(\frac{2^{n} \pi^{2}}{6(N-1)}\right)^{\frac{1}{n+1}}$, i.e. $\left.\delta^{n+1}=\frac{2^{n} \pi^{2}}{6(N-1)}\right)$

$$
=\frac{\omega_{1}\left(f^{(n)},\left(\frac{2^{n} \pi^{2}}{6(N-1)}\right)^{\frac{1}{n+1}}\right)}{n !}\left[\frac{2^{n-1} \pi^{2}}{6(N-1)}+\frac{1}{(n+1)}\left(\frac{2^{n} \pi^{2}}{6(N-1)}\right)^{\frac{n}{n+1}}\right],
$$

proving the claim.

The case $n=1$ follows:

Corollary 2. Let $f \in C^{1}\left([-1,1], \mathbb{R}_{+}\right), x \in[-1,1], f^{\prime}(x)=0$. Here $N \in \mathbb{N}, N \geq 3$ is odd. Then

$$
\begin{aligned}
& \left|L_{N}^{(M)}(f)(x)-f(x)\right| \\
& \quad \leq \omega_{1}\left(f^{\prime}, \frac{\pi}{\sqrt{3(N-1)}}\right)\left[\frac{\pi^{2}}{6(N-1)}+\frac{\pi}{2 \sqrt{3(N-1)}}\right] .
\end{aligned}
$$

By (36) and/or (38), we get that $L_{N}^{(M)}(f)(x) \rightarrow f(x)$, as $N \rightarrow \infty$.

We make

Remark 4. Here we compare (38) to (33), and we prove that (38) gives better estimates and speeds than (33). We observe that

$$
\frac{\pi}{\sqrt{3(N-1)}}\left[\frac{\pi^{2}}{6(N-1)}+\frac{\pi}{2 \sqrt{3(N-1)}}\right] \leq \frac{4}{N-1}
$$




$$
\begin{gathered}
\Leftrightarrow \\
\frac{1}{\sqrt{3(N-1)}} \leq \frac{24-\pi^{2}}{\pi^{3}},
\end{gathered}
$$

true for large enough $N \geq 3$ odd.

Remark 5. Here we compare (36) to (33), and we prove that (36) gives better estimates and speeds that (33). We see that

$$
\begin{gathered}
\frac{1}{n !}\left(\frac{2^{n} \pi^{2}}{6(N-1)}\right)^{\frac{1}{n+1}}\left[\frac{2^{n-1} \pi^{2}}{6(N-1)}+\frac{1}{(n+1)}\left(\frac{2^{n} \pi^{2}}{6(N-1)}\right)^{\frac{n}{n+1}}\right] \\
\leq \frac{4}{N-1} \\
\Leftrightarrow \\
\frac{1}{N-1} \leq \frac{3}{2^{n-1} \pi^{2}}\left(\frac{24(n+1) !-2^{n} \pi^{2}}{(n+1) 2^{n-1} \pi^{2}}\right)^{n+1},
\end{gathered}
$$

true for large enough $N$ (odd) $\geq 3$.

We continue with

Remark 6. From [3], p. 297, we have: Let $f \in C\left([-1,1], \mathbb{R}_{+}\right), N \geq 4$, $N \in \mathbb{N}, N$ even. Then

$$
\left|L_{N}^{(M)}(f)(x)-f(x)\right| \leq 28 \omega_{1}\left(f, \frac{1}{N-1}\right), \forall x \in[-1,1] .
$$

From [3], p. 298, we get

$$
L_{N}^{(M)}(|\cdot-x|)(x) \leq \frac{4 \pi^{2}}{3(N-1)}=\frac{2^{2} \pi^{2}}{3(N-1)}, \quad \forall x \in(-1,1) .
$$

Hence $(m \in \mathbb{N})$

$$
L_{N}^{(M)}\left(|\cdot-x|^{m}\right)(x) \leq \frac{2^{m+1} \pi^{2}}{3(N-1)}, \quad \forall x \in(-1,1) .
$$

We present

Theorem 4. Let $f \in C^{n}\left([-1,1], \mathbb{R}_{+}\right), n \in \mathbb{N}, x \in[-1,1], f^{(i)}(x)=0$, $i=1, \ldots, n$. Here $N \in \mathbb{N}, N \geq 4, N$ is even. Then

$$
\begin{aligned}
(46) \mid & L_{N}^{(M)}(f)(x)-f(x) \mid \\
& \leq \frac{\omega_{1}\left(f^{(n)},\left(\frac{2^{n+2} \pi^{2}}{3(N-1)}\right)^{\frac{1}{n+1}}\right)}{n !}\left[\frac{2^{n+1} \pi^{2}}{3(N-1)}+\frac{1}{(n+1)}\left(\frac{2^{n+2} \pi^{2}}{3(N-1)}\right)^{\frac{n}{n+1}}\right] .
\end{aligned}
$$


Proof. When $x= \pm 1$, the left hand side of (46) is zero, thus (46) is trivially true.

Let now $x \in(-1,1)$, by Theorem 1 and (16), (45), we obtain

$$
\begin{aligned}
& \left|L_{N}^{(M)}(f)(x)-f(x)\right| \\
& \quad \leq \frac{\omega_{1}\left(f^{(n)}, \delta\right)}{n !}\left[\frac{2^{n+1} \pi^{2}}{3(N-1)}+\frac{1}{(n+1) \delta} \frac{2^{n+2} \pi^{2}}{3(N-1)}\right]
\end{aligned}
$$

$\left(\right.$ setting $\delta:=\left(\frac{2^{n+2} \pi^{2}}{3(N-1)}\right)^{\frac{1}{n+1}}$, i.e. $\left.\delta^{n+1}=\frac{2^{n+2} \pi^{2}}{3(N-1)}\right)$

$$
=\frac{\omega_{1}\left(f^{(n)},\left(\frac{2^{n+2} \pi^{2}}{3(N-1)}\right)^{\frac{1}{n+1}}\right)}{n !}\left[\frac{2^{n+1} \pi^{2}}{3(N-1)}+\frac{1}{(n+1)}\left(\frac{2^{n+2} \pi^{2}}{3(N-1)}\right)^{\frac{n}{n+1}}\right],
$$

proving the claim.

The case $n=1$ follows:

Corollary 3. Let $f \in C^{1}\left([-1,1], \mathbb{R}_{+}\right), x \in[-1,1], f^{\prime}(x)=0$. Here $N \in \mathbb{N}, N \geq 4, N$ is even. Then

$$
\begin{aligned}
& \left|L_{N}^{(M)}(f)(x)-f(x)\right| \\
& \quad \leq \omega_{1}\left(f^{\prime}, \frac{2 \pi \sqrt{2}}{\sqrt{3(N-1)}}\right)\left[\frac{4 \pi^{2}}{3(N-1)}+\frac{\pi \sqrt{2}}{\sqrt{3(N-1)}}\right] .
\end{aligned}
$$

By (46) and/or (48), we get that $L_{N}^{(M)}(f)(x) \rightarrow f(x)$, as $N \rightarrow \infty$.

We make

Remark 7. Here we compare (48) to (43). We prove that (48) gives better estimates and speeds that (43). Indeed we have

$$
\begin{gathered}
\frac{2 \pi \sqrt{2}}{\sqrt{3(N-1)}}\left[\frac{4 \pi^{2}}{3(N-1)}+\frac{\pi \sqrt{2}}{\sqrt{3(N-1)}}\right] \leq \frac{28}{N-1} \\
\Leftrightarrow \\
\frac{1}{N-1} \leq \frac{3}{8 \pi^{2}}\left(\frac{42-2 \pi^{2}}{2 \pi^{2}}\right)^{2},
\end{gathered}
$$

true for large enough $N \geq 4$, even.

We make 
Remark 8. Here we compare (46) to (43). We prove that (46) gives better estimates and speeds that (43). We observe that

$$
\begin{gathered}
\frac{1}{n !}\left(\frac{2^{n+2} \pi^{2}}{3(N-1)}\right)^{\frac{1}{n+1}}\left[\frac{2^{n+1} \pi^{2}}{3(N-1)}+\frac{1}{(n+1)}\left(\frac{2^{n+2} \pi^{2}}{3(N-1)}\right)^{\frac{n}{n+1}}\right] \\
\leq \frac{28}{N-1} \\
\Leftrightarrow \\
\frac{1}{N-1} \leq\left(\frac{3}{2^{n+2} \pi^{2}}\right)\left[\frac{42(n+1) !-2^{n+1} \pi^{2}}{(n+1) 2^{n} \pi^{2}}\right]^{n+1},
\end{gathered}
$$

true for large enough $N \geq 4$. $N$ even.

We continue with

Remark 9. The sampling truncated linear operators (see [3], p. 7) are defined by

$$
W_{N}(f)(x)=\sum_{k=0}^{N} \frac{\sin (N x-k \pi)}{N x-k \pi} f\left(\frac{k \pi}{N}\right), \forall x \in[0, \pi],
$$

and

$$
T_{N}(f)(x)=\sum_{k=0}^{N} \frac{\sin ^{2}(N x-k \pi)}{(N x-k \pi)^{2}} f\left(\frac{k \pi}{N}\right),
$$

$\forall x \in[0, \pi] ; f \in C([0, \pi], \mathbb{R})$ and they are used as approximators.

Here we deal with the Max-product truncated sampling operators (see [3], p. 13) defined by

$$
W_{N}^{(M)}(f)(x)=\frac{\bigvee_{k=0}^{N} \frac{\sin (N x-k \pi)}{N x-k \pi} f\left(\frac{k \pi}{N}\right)}{\bigvee_{k=0}^{N} \frac{\sin (N x-k \pi)}{N x-k \pi}}, x \in[0, \pi],
$$

$f:[0, \pi] \rightarrow \mathbb{R}_{+}$, continuous, and

$$
T_{N}^{(M)}(f)(x)=\frac{\bigvee_{k=0}^{N} \frac{\sin ^{2}(N x-k \pi)}{(N x-k \pi)^{2}} f\left(\frac{k \pi}{N}\right)}{\bigvee_{k=0}^{N} \frac{\sin ^{2}(N x-k \pi)}{(N x-k \pi)^{2}}}, x \in[0, \pi],
$$

$f:[0, \pi] \rightarrow \mathbb{R}_{+}$, continuous.

Following [3], p. 343, and making the convention $\frac{\sin (0)}{0}=1$ and denoting $s_{N, k}(x)=\frac{\sin (N x-k \pi)}{N x-k \pi}$, we get that $s_{N, k}\left(\frac{k \pi}{N}\right)=1$, and $s_{N, k}\left(\frac{j \pi}{N}\right)=0$, if $k \neq j$, furthermore $W_{N}^{(M)}(f)\left(\frac{j \pi}{N}\right)=f\left(\frac{j \pi}{N}\right)$, for all $j \in\{0, \ldots, N\}$. 
Clearly $W_{N}^{(M)}(f)$ is a well-defined function for all $x \in[0, \pi]$, and it is continuous on $[0, \pi]$, also $W_{N}^{(M)}(1)=1$.

By [3], p. 344, $W_{N}^{(M)}$ are positive sublinear operators.

Call $I_{N}^{+}(x)=\left\{k \in\{0,1, \ldots, N\} ; s_{N, k}(x)>0\right\}$, and set $x_{N, k}:=\frac{k \pi}{N}, k \in$ $\{0,1, \ldots, N\}$.

We see that

$$
W_{N}^{(M)}(f)(x)=\frac{\bigvee_{k \in I_{N}^{+}(x)} s_{N, k}(x) f\left(x_{N, k}\right)}{\bigvee_{k \in I_{N}^{+}(x)} s_{N, k}(x)}
$$

We call

$$
\begin{aligned}
F_{N}(x) & :=W_{N}^{(M)}(|\cdot-x|)(x)=\frac{\bigvee_{k=0}^{N} s_{N, k}(x)\left|x_{N, k}-x\right|}{\bigvee_{k=0}^{N} s_{N, k}(x)} \\
& =\frac{\bigvee_{k \in I_{N}^{+}(x)} s_{N, k}(x)\left|x_{N, k}-x\right|}{\bigvee_{k \in I_{N}^{+}(x)} s_{N, k}(x)} .
\end{aligned}
$$

By Theorem $8.2 .8([3]$, p. 345$)$ we get: Let $f \in C\left([0, \pi], \mathbb{R}_{+}\right)$. Then

$$
\left|W_{N}^{(M)}(f)(x)-f(x)\right| \leq 4 \omega_{1}\left(f, \frac{1}{N}\right)_{[0, \pi]}, \quad \forall N \in \mathbb{N}, x \in[0, \pi] .
$$

We have that $([3]$, p. 346)

$$
F_{N}(x) \leq \frac{\pi}{2 N}
$$

Notice also $\left|x_{N, k}-x\right| \leq \pi, \forall x \in[0, \pi]$.

Therefore $(m \in \mathbb{N})$ it holds

$$
W_{N}^{(M)}\left(|\cdot-x|^{m}\right)(x) \leq \frac{\pi^{m-1} \pi}{2 N}=\frac{\pi^{m}}{2 N} .
$$

We present

Theorem 5. Let $f \in C^{n}\left([0, \pi], \mathbb{R}_{+}\right), x \in[0, \pi]$ fixed, $f^{(i)}(x)=0$, $i=1, \ldots, n$. Then

$$
\begin{gathered}
\left|W_{N}^{(M)}(f)(x)-f(x)\right| \leq \frac{\omega_{1}\left(f^{(n)},\left(\frac{\pi^{n+1}}{2 N}\right)^{\frac{1}{n+1}}\right)}{n !}\left[\frac{\pi^{n}}{2 N}+\frac{\left(\frac{\pi^{n+1}}{2 N}\right)^{\frac{n}{n+1}}}{n+1}\right] \\
=\frac{1}{n !} \omega_{1}\left(f^{(n)}, \frac{\pi}{\sqrt[n+1]{2 N}}\right)\left[\frac{\pi^{n}}{2 N}+\frac{1}{n+1}\left(\frac{\pi}{\sqrt[n+1]{2 N}}\right)^{n}\right] .
\end{gathered}
$$


Proof. Using Theorem 1, (16) and (61), we get

$$
\left|W_{N}^{(M)}(f)(x)-f(x)\right| \leq \frac{\omega_{1}\left(f^{(n)}, \delta\right)}{n !}\left[\frac{\pi^{n}}{2 N}+\frac{1}{(n+1) \delta}\left(\frac{\pi^{n+1}}{2 N}\right)\right]
$$

( $\operatorname{choosing} \delta:=\left(\frac{\pi^{n+1}}{2 N}\right)^{\frac{1}{n+1}}$, i.e. $\delta^{n+1}=\frac{\pi^{n+1}}{2 N}$ )

$$
=\frac{1}{n !} \omega_{1}\left(f^{(n)},\left(\frac{\pi^{n+1}}{2 N}\right)^{\frac{1}{n+1}}\right)\left[\frac{\pi^{n}}{2 N}+\frac{1}{(n+1)}\left(\frac{\pi^{n+1}}{2 N}\right)^{\frac{n}{n+1}}\right],
$$

proving the claim.

The case $n=1$ follows:

Corollary 4. Let $f \in C^{1}\left([0, \pi], \mathbb{R}_{+}\right), x \in[0, \pi]$ fixed, $f^{\prime}(x)=0$. Then

$$
\left|W_{N}^{(M)}(f)(x)-f(x)\right| \leq \omega_{1}\left(f^{\prime}, \frac{\pi}{\sqrt{2 N}}\right)\left[\frac{\pi}{2 N}+\frac{\pi}{2 \sqrt{2 N}}\right] .
$$

By (62)-(63) and/or (65), we get that $W_{N}^{(M)}(f)(x) \rightarrow f(x)$, as $N \rightarrow+\infty$.

We make

Remark 10. Here we compare (65) to (59) and we prove that (65) gives better estimates and speeds that (59). Indeed we have

$$
\begin{gathered}
\frac{\pi}{\sqrt{2 N}}\left(\frac{\pi}{2 N}+\frac{\pi}{2 \sqrt{2 N}}\right) \leq \frac{4}{N} \\
\Leftrightarrow \\
\frac{1}{\sqrt{2 N}} \leq \frac{16-\pi^{2}}{2 \pi^{2}},
\end{gathered}
$$

true for large enough $N \in \mathbb{N}$.

We also make

Remark 11. Here we compare (62)-(63) to (59), and we prove that (62)-(63) gives better estimates and speeds that (59). We observe that

$$
\begin{aligned}
& \frac{1}{n !}\left(\frac{\pi^{n+1}}{2 N}\right)^{\frac{1}{n+1}}\left[\frac{\pi^{n}}{2 N}+\frac{\left(\frac{\pi^{n+1}}{2 N}\right)^{\frac{n}{n+1}}}{(n+1)}\right] \leq \frac{4}{N} \\
& \Leftrightarrow \\
& \frac{1}{\sqrt[n+1]{2 N}} \leq \frac{8(n+1) !-\pi^{n+1}}{(n+1) \pi^{n+1}},
\end{aligned}
$$

true for large enough $N \in \mathbb{N}$.

Notice here that $8(n+1) !-\pi^{n+1}>0, \forall n \in \mathbb{N}$. 
We continue with

Remark 12. Here we study $T_{N}(f)(x)$, see (54).

By Theorem 8.2.13, [3], p. 352, we get: Let $f \in C\left([0, \pi], \mathbb{R}_{+}\right)$, then

$$
\left|T_{N}^{(M)}(f)(x)-f(x)\right| \leq 4 \omega_{1}\left(f, \frac{1}{N}\right)_{[0, \pi]}, \quad \forall N \in \mathbb{N}, x \in[0, \pi] .
$$

By [3], p. 352, we get

$$
T_{N}^{(M)}(|\cdot-x|)(x) \leq \frac{\pi}{2 N}
$$

hence $(m \in \mathbb{N})$ we find

$$
T_{N}^{(M)}\left(|\cdot-x|^{m}\right)(x) \leq \frac{\pi^{m}}{2 N}
$$

Here again $x_{N, k}=\frac{k \pi}{N}, k \in\{0,1, \ldots, N\}$.

The operators $T_{N}^{(M)}$ are positive sublinear operators, mapping $C\left([0, \pi], \mathbb{R}_{+}\right)$ into itself, and $T_{N}^{(M)}(1)=1$. So we can apply again Theorem 1 . We obtain the same results as before with $W_{N}^{(M)}$, we state them:

Theorem 6. Let $f \in C^{1}\left([0, \pi], \mathbb{R}_{+}\right), x \in[0, \pi]$ fixed, $f^{\prime}(x)=0$. Then

$$
\left|T_{N}^{(M)}(f)(x)-f(x)\right| \leq \omega_{1}\left(f^{\prime}, \frac{\pi}{\sqrt{2 N}}\right)\left[\frac{\pi}{2 N}+\frac{\pi}{2 \sqrt{2 N}}\right], \quad \forall N \in \mathbb{N}
$$

Theorem 7. Let $f \in C^{n}\left([0, \pi], \mathbb{R}_{+}\right), x \in[0, \pi]$ fixed, $f^{(i)}(x)=0$, $i=1, \ldots, n$. Then

$$
\begin{aligned}
& \left|T_{N}^{(M)}(f)(x)-f(x)\right| \\
& \quad \leq \frac{\omega_{1}\left(f^{(n)}, \frac{\pi}{\sqrt[n+1]{2 N}}\right)}{n !}\left[\frac{\pi^{n}}{2 N}+\frac{\left(\frac{\pi}{\sqrt[n+1]{2 N}}\right)^{n}}{n+1}\right], \quad \forall N \in \mathbb{N} .
\end{aligned}
$$

Clearly (73), (74) can perform better than (70), the same study as for $W_{N}^{(M)}$.

Furthermore we derive $T_{N}^{(M)}(f)(x) \rightarrow f(x)$, as $N \rightarrow+\infty$.

We continue with

Remark 13. Let $b: \mathbb{R} \rightarrow \mathbb{R}_{+}$be a centered (it takes a global maximum at 0 ) bell-shaped function, with compact support $[-T, T], T>0$ (that is $b(x)>0$ for all $x \in(-T, T))$ and $I=\int_{-T}^{T} b(x) d x>0$. 
The Cardaliaguet-Euvrard neural network operators are defined by (see [2])

$$
C_{N, \alpha}(f)(x)=\sum_{k=-N^{2}}^{N^{2}} \frac{f\left(\frac{k}{N}\right)}{I N^{1-\alpha}} b\left(N^{1-\alpha}\left(x-\frac{k}{N}\right)\right),
$$

$0<\alpha<1, N \in \mathbb{N}$ and $f: \mathbb{R} \rightarrow \mathbb{R}$ is continuous and bounded or uniformly continuous on $\mathbb{R}$.

$C B(\mathbb{R})$ denotes the continuous and bounded function on $\mathbb{R}$, and

$$
C B_{+}(\mathbb{R})=\{f: \mathbb{R} \rightarrow[0, \infty) ; f \in C B(\mathbb{R})\} .
$$

The corresponding max-product Cardaliaguet-Euvrard neural network operators will be given by

$$
C_{N, \alpha}^{(M)}(f)(x)=\frac{\bigvee_{k=-N^{2}}^{N^{2}} b\left(N^{1-\alpha}\left(x-\frac{k}{N}\right)\right) f\left(\frac{k}{N}\right)}{\bigvee_{k=-N^{2}}^{N^{2}} b\left(N^{1-\alpha}\left(x-\frac{k}{N}\right)\right)},
$$

$f \in \mathbb{R}, f \in C B_{+}(\mathbb{R})$, see also [2].

Next we follow [2].

For any $x \in \mathbb{R}$, denoting

$$
J_{T, N}(x)=\left\{k \in \mathbb{Z} ;-N^{2} \leq k \leq N^{2}, N^{1-\alpha}\left(x-\frac{k}{N}\right) \in(-T, T)\right\},
$$

we can write

$$
C_{N, \alpha}^{(M)}(f)(x)=\frac{\bigvee_{k \in J_{T, N}(x)} b\left(N^{1-\alpha}\left(x-\frac{k}{N}\right)\right) f\left(\frac{k}{N}\right)}{\bigvee_{k \in J_{T, N}(x)} b\left(N^{1-\alpha}\left(x-\frac{k}{N}\right)\right)},
$$

$x \in \mathbb{R}, N>\max \left\{T+|x|, T^{-\frac{1}{\alpha}}\right\}$, where $J_{T, N}(x) \neq \emptyset$. Indeed, we have $\bigvee_{k \in J_{T, N}(x)} b\left(N^{1-\alpha}\left(x-\frac{k}{N}\right)\right)>0, \forall x \in \mathbb{R}$ and $N>\max \left\{T+|x|, T^{-\frac{1}{\alpha}}\right\}$.

We have that $C_{N, \alpha}^{(M)}(1)(x)=1, \forall x \in \mathbb{R}$ and $N>\max \left\{T+|x|, T^{-\frac{1}{\alpha}}\right\}$.

See in [2] there: Lemma 2.1, Corollary 2.2 and Remarks.

We need

Theorem 8 ([2]). Let $b(x)$ be a centered bell-shaped function, continuous and with compact support $[-T, T], T>0,0<\alpha<1$ and $C_{N, \alpha}^{(M)}$ be defined as in (76).

(i) If $|f(x)| \leq c$ for all $x \in \mathbb{R}$ then $\left|C_{N, \alpha}^{(M)}(f)(x)\right| \leq c$, for all $x \in \mathbb{R}$ and $N>\max \left\{T+|x|, T^{-\frac{1}{\alpha}}\right\}$ and $C_{N, \alpha}^{(M)}(f)(x)$ is continuous at any point $x \in \mathbb{R}$, for all $N>\max \left\{T+|x|, T^{-\frac{1}{\alpha}}\right\}$; 
(ii) If $f, g \in C B_{+}(\mathbb{R})$ satisfy $f(x) \leq g(x)$ for all $x \in \mathbb{R}$, then $C_{N, \alpha}^{(M)}(f)(x) \leq$ $C_{N, \alpha}^{(M)}(g)(x)$ for all $x \in \mathbb{R}$ and $N>\max \left\{T+|x|, T^{-\frac{1}{\alpha}}\right\}$;

$($ iii $) C_{N, \alpha}^{(M)}(f+g)(x) \leq C_{N, \alpha}^{(M)}(f)(x)+C_{N, \alpha}^{(M)}(g)(x)$ for all $f, g \in C B_{+}(\mathbb{R})$, $x \in \mathbb{R}$ and $N>\max \left\{T+|x|, T^{-\frac{1}{\alpha}}\right\}$; have

(iv) For all $f, g \in C B_{+}(\mathbb{R}), x \in \mathbb{R}$ and $N>\max \left\{T+|x|, T^{-\frac{1}{\alpha}}\right\}$, we

$$
\left|C_{N, \alpha}^{(M)}(f)(x)-C_{N, \alpha}^{(M)}(g)(x)\right| \leq C_{N, \alpha}^{(M)}(|f-g|)(x) ;
$$

$(v) C_{N, \alpha}^{(M)}$ is positive homogeneous, that is $C_{N, \alpha}^{(M)}(\lambda f)(x)=\lambda C_{N, \alpha}^{(M)}(f)(x)$ for all $\lambda \geq 0, x \in \mathbb{R}, N>\max \left\{T+|x|, T^{-\frac{1}{\alpha}}\right\}$ and $f \in C B_{+}(\mathbb{R})$.

We make

Remark 14. We have

$$
\begin{aligned}
E_{N, \alpha}(x) & :=C_{N, \alpha}^{(M)}(|\cdot-x|)(x) \\
& =\frac{\bigvee_{k \in J_{T, N}(x)} b\left(N^{1-\alpha}\left(x-\frac{k}{N}\right)\right)\left|x-\frac{k}{N}\right|}{\bigvee_{k \in J_{T, N}(x)} b\left(N^{1-\alpha}\left(x-\frac{k}{N}\right)\right)},
\end{aligned}
$$

$\forall x \in \mathbb{R}$, and $N>\max \left\{T+|x|, T^{-\frac{1}{\alpha}}\right\}$.

By $(77), C_{N, \alpha}^{(M)}$ satisfies

$$
C_{N, \alpha}^{(M)}(f \vee g)(x)=C_{N, \alpha}^{(M)}(f)(x) \vee C_{N, \alpha}^{(M)}(g)(x),
$$

$\forall f, g \in C B_{+}(\mathbb{R}), x \in \mathbb{R}, N>\max \left\{T+|x|, T^{-\frac{1}{\alpha}}\right\}$.

Notice that

$$
\bigvee_{k \in J_{T, N}(x)} b\left(N^{1-\alpha}\left(x-\frac{k}{N}\right)\right)=\bigvee_{k=-N^{2}}^{N^{2}} b\left(N^{1-\alpha}\left(x-\frac{k}{N}\right)\right) .
$$

By [2], Lemma 3.1 there, we have: Let $b(x)$ be a centered bell-shaped function, continuous and with compact support $[-T, T], T>0$ and $0<\alpha<1$. Then for any $j \in \mathbb{Z}$ with $-N^{2} \leq j \leq N^{2}$, all $x \in\left[\frac{j}{N}, \frac{j+1}{N}\right]$ and $N>$ $\max \left\{T+|x|, T^{-\frac{1}{\alpha}}\right\}$, we have

$$
\begin{aligned}
& \bigvee_{k=-N^{2}}^{N^{2}} b\left(N^{1-\alpha}\left(x-\frac{k}{N}\right)\right) \\
& =\max \left\{b\left(N^{1-\alpha}\left(x-\frac{j}{N}\right)\right), b\left(N^{1-\alpha}\left(x-\frac{j+1}{N}\right)\right)\right\}>0 .
\end{aligned}
$$


Lemma $3.1([2])$, is valid only for all $x \in[-N, N]$.

We mention from [2] the following:

Theorem $9([2])$. Let $b(x)$ be a centered bell-shaped function, continuous and with compact support $[-T, T], T>0$ and $0<\alpha<1$. In addition, suppose that the following requirements are fulfilled:

(i) There exist $0<m_{1} \leq M_{1}<\infty$ such that $m_{1}(T-x) \leq b(x) \leq$ $M_{1}(T-x), \forall x \in[0, T]$;

(ii) There exist $0<m_{2} \leq M_{2}<\infty$ such that $m_{2}(x+T) \leq b(x) \leq$ $M_{2}(x+T), \forall x \in[-T, 0]$.

Then for all $f \in C B_{+}(\mathbb{R}), x \in \mathbb{R}$ and for all $N \in \mathbb{N}$ satisying $N>$ $\max \left\{T+|x|,\left(\frac{2}{T}\right)^{\frac{1}{\alpha}}\right\}$, we have the estimate

$$
\left|C_{N, \alpha}^{(M)}(f)(x)-f(x)\right| \leq c \omega_{1}\left(f, N^{\alpha-1}\right)_{\mathbb{R}},
$$

where

$$
c:=2\left(\max \left\{\frac{T M_{2}}{2 m_{2}}, \frac{T M_{1}}{2 m_{1}}\right\}+1\right)
$$

and

$$
\omega_{1}(f, \delta)_{\mathbb{R}}:=\sup _{\substack{x, y \in \mathbb{R}: \\|x-y| \leq \delta}}|f(x)-f(y)|
$$

We make

Remark 15. In [2], was proved that

$$
\begin{gathered}
E_{N, \alpha}(x) \leq \max \left\{\frac{T M_{2}}{2 m_{2}}, \frac{T M_{1}}{2 m_{1}}\right\} N^{\alpha-1}, \\
\forall N>\max \left\{T+|x|,\left(\frac{2}{T}\right)^{\frac{1}{\alpha}}\right\} .
\end{gathered}
$$

That is

$$
\begin{aligned}
& C_{N, \alpha}^{(M)}(|\cdot-x|)(x) \leq \max \left\{\frac{T M_{2}}{2 m_{2}}, \frac{T M_{1}}{2 m_{1}}\right\} N^{\alpha-1}, \\
& \forall N>\max \left\{T+|x|,\left(\frac{2}{T}\right)^{\frac{1}{\alpha}}\right\} .
\end{aligned}
$$

From (78) we have that $\left|x-\frac{k}{N}\right| \leq \frac{T}{N^{1-\alpha}}$. 
Hence $(m \in \mathbb{N})\left(\forall x \in \mathbb{R}\right.$ and $\left.N>\max \left\{T+|x|,\left(\frac{2}{T}\right)^{\frac{1}{\alpha}}\right\}\right)$

$$
\begin{aligned}
C_{N, \alpha}^{(M)}\left(|\cdot-x|^{m}\right)(x) & =\frac{\bigvee_{k \in J_{T, N}(x)} b\left(N^{1-\alpha}\left(x-\frac{k}{N}\right)\right)\left|x-\frac{k}{N}\right|^{m}}{\bigvee_{k \in J_{T, N}(x)} b\left(N^{1-\alpha}\left(x-\frac{k}{N}\right)\right)} \\
& \leq\left(\frac{T}{N^{1-\alpha}}\right)^{m-1} \max \left\{\frac{T M_{2}}{2 m_{2}}, \frac{T M_{1}}{2 m_{1}}\right\} N^{\alpha-1}, \\
\forall N & >\max \left\{T+|x|,\left(\frac{2}{T}\right)^{\frac{1}{\alpha}}\right\} .
\end{aligned}
$$

Then $(m \in \mathbb{N})$ it holds

$$
\begin{gathered}
C_{N, \alpha}^{(M)}\left(|\cdot-x|^{m}\right)(x) \leq T^{m-1} \max \left\{\frac{T M_{2}}{2 m_{2}}, \frac{T M_{1}}{2 m_{1}}\right\} \frac{1}{N^{m(1-\alpha)}}, \\
\forall N>\max \left\{T+|x|,\left(\frac{2}{T}\right)^{\frac{1}{\alpha}}\right\} .
\end{gathered}
$$

Call

$$
\lambda:=\max \left\{\frac{T M_{2}}{2 m_{2}}, \frac{T M_{1}}{2 m_{1}}\right\}>0 .
$$

Consequently $(m \in \mathbb{N})$ we derive

$$
C_{N, \alpha}^{(M)}\left(|\cdot-x|^{m}\right)(x) \leq \frac{\lambda T^{m-1}}{N^{m(1-\alpha)}}, \quad \forall N>\max \left\{T+|x|,\left(\frac{2}{T}\right)^{\frac{1}{\alpha}}\right\}
$$

We need

Theorem 10. Let $b(x)$ be a centered bell-shaped function, continuous and with compact support $[-T, T], T>0,0<\alpha<1$ and $C_{N, \alpha}^{(M)}$ be defined as in (76).

Let $f \in C B_{+}^{n}(\mathbb{R}), n \in \mathbb{N}$. Let $x \in \mathbb{R}: f^{(i)}(x)=0, i=1, \ldots, n$, and $\delta>0$. Then

$$
\begin{aligned}
& \left|C_{N, \alpha}^{(M)}(f)(x)-f(x)\right| \\
& \quad \leq \frac{\omega_{1}\left(f^{(n)}, \delta\right)_{\mathbb{R}}}{n !}\left[C_{N, \alpha}^{(M)}\left(|\cdot-x|^{n}\right)(x)+\frac{C_{N, \alpha}^{(M)}\left(|\cdot-x|^{n+1}\right)(x)}{(n+1) \delta}\right],
\end{aligned}
$$

$\forall N \in \mathbb{N}: N>\max \left\{T+|x|, T^{-\frac{1}{\alpha}}\right\}$ 
Proof. By [1], we get that

$$
|f(x)-f(y)| \leq \frac{\omega_{1}\left(f^{(n)}, \delta\right)_{\mathbb{R}}}{n !}\left[|x-y|^{n}+\frac{|x-y|^{n+1}}{(n+1) \delta}\right],
$$

$\forall y \in \mathbb{R}, \delta>0$. Using Theorem 8 and $C_{N, \alpha}^{(M)}(1)=1$, we get

$$
\begin{aligned}
& \left|C_{N, \alpha}^{(M)}(f)(x)-f(x)\right| \leq C_{N, \alpha}^{(M)}(|f(\cdot)-f(x)|)(x) \\
& \quad \leq \frac{\omega_{1}\left(f^{(n)}, \delta\right)_{\mathbb{R}}}{n !}\left[C_{N, \alpha}^{(M)}\left(|\cdot-x|^{n}\right)(x)+\frac{C_{N, \alpha}^{(M)}\left(|\cdot-x|^{n+1}\right)(x)}{(n+1) \delta}\right],
\end{aligned}
$$

$\forall N \in \mathbb{N}: N>\max \left\{T+|x|, T^{-\frac{1}{\alpha}}\right\}$.

We give

Theorem 11. Same assumptions as in Theorem 9. Let $f \in C B_{+}^{n}(\mathbb{R})$, $n \in \mathbb{N}, x \in \mathbb{R}: f^{(i)}(x)=0, i=1, \ldots, n$. Then

$$
\begin{aligned}
\mid C_{N, \alpha}^{(M)}(f)(x) & -f(x) \mid \leq \frac{\omega_{1}\left(f^{(n)},\left(\frac{\lambda T^{n}}{N^{(n+1)(1-\alpha)}}\right)^{\frac{1}{n+1}}\right)_{\mathbb{R}}}{n !} \\
\times & {\left[\frac{\lambda T^{n-1}}{N^{n(1-\alpha)}}+\frac{1}{(n+1)}\left(\frac{\lambda T^{n}}{N^{(n+1)(1-\alpha)}}\right)^{\frac{n}{n+1}}\right], }
\end{aligned}
$$

$\forall N>\max \left\{T+|x|,\left(\frac{2}{T}\right)^{\frac{1}{\alpha}}\right\}$.

Proof. We use (88) and we choose

$$
\delta:=\left(\frac{\lambda T^{n}}{N^{(n+1)(1-\alpha)}}\right)^{\frac{1}{n+1}}
$$

i.e. $\delta^{n+1}=\frac{\lambda T^{n}}{N^{(n+1)(1-\alpha)}}$. Hence

$$
\begin{aligned}
\mid C_{N, \alpha}^{(M)}(f)(x) & -\left.f(x)\right|^{((87),(88))} \frac{1}{n !} \omega_{1}\left(f^{(n)},\left(\frac{\lambda T^{n}}{N^{(n+1)(1-\alpha)}}\right)^{\frac{1}{n+1}}\right)_{\mathbb{R}} \\
& \times\left[\frac{\lambda T^{n-1}}{N^{n(1-\alpha)}}+\frac{1}{(n+1) \delta} \frac{\lambda T^{n}}{N^{(n+1)(1-\alpha)}}\right] \\
= & \frac{1}{n !} \omega_{1}\left(f^{(n)},\left(\frac{\lambda T^{n}}{N^{(n+1)(1-\alpha)}}\right)^{\frac{1}{n+1}}\right)_{\mathbb{R}} \\
& \times\left[\frac{\lambda T^{n-1}}{N^{n(1-\alpha)}}+\frac{1}{(n+1)}\left(\frac{\lambda T^{n}}{N^{(n+1)(1-\alpha)}}\right)^{\frac{n}{n+1}}\right],
\end{aligned}
$$


$\forall N>\max \left\{T+|x|,\left(\frac{2}{T}\right)^{\frac{1}{\alpha}}\right\}$, proving the claim.

It follows the case $n=1$.

Corollary 5. Same assumptions as in Theorem 9. Let $f \in C B_{+}^{1}(\mathbb{R})$, $x \in \mathbb{R}: f^{\prime}(x)=0$. Then

$$
\begin{aligned}
& \left|C_{N, \alpha}^{(M)}(f)(x)-f(x)\right| \\
& \quad \leq \omega_{1}\left(f^{\prime}, \sqrt{\frac{\lambda T}{N^{2(1-\alpha)}}}\right)_{\mathbb{R}}\left[\frac{\lambda}{N^{1-\alpha}}+\frac{1}{2} \sqrt{\frac{\lambda T}{N^{2(1-\alpha)}}}\right],
\end{aligned}
$$

$\forall N>\max \left\{T+|x|,\left(\frac{2}{T}\right)^{\frac{1}{\alpha}}\right\}$.

By (91) and/or (94) we get that $C_{N, \alpha}^{(M)}(f)(x) \rightarrow f(x)$, as $N \rightarrow+\infty$.

We make

Remark 16. We prove that (94) performs better than (81).

Indeed we have that

$$
\begin{gathered}
\sqrt{\frac{\lambda T}{N^{2(1-\alpha)}}\left[\frac{\lambda}{N^{1-\alpha}}+\right.}+\frac{1}{2} \sqrt{\left.\frac{\lambda T}{N^{2(1-\alpha)}}\right] \leq \frac{2(\lambda+1)}{N^{1-\alpha}},} \\
\Leftrightarrow \\
\frac{1}{N^{(1-\alpha)}} \leq \frac{2(\lambda+1)}{\lambda\left(\sqrt{\lambda T}+\frac{T}{2}\right)},
\end{gathered}
$$

true $\forall N>\max \left\{T+|x|,\left(\frac{2}{T}\right)^{\frac{1}{\alpha}}\right\}$, large enough.

We also make

Remark 17. We prove that (91) performs better than (81). We observe that

$$
\begin{aligned}
\frac{1}{n !}\left(\frac{\lambda T^{n}}{\left.N^{(n+1)(1-\alpha)}\right)^{\frac{1}{n+1}}}\right. & {\left[\frac{\lambda T^{n-1}}{N^{n(1-\alpha)}}+\frac{1}{(n+1)}\left(\frac{\lambda T^{n}}{N^{(n+1)(1-\alpha)}}\right)^{\frac{n}{n+1}}\right] } \\
\leq \frac{2(\lambda+1)}{N^{1-\alpha}} & \Leftrightarrow
\end{aligned}
$$

$$
\frac{1}{N^{n(1-\alpha)}} \leq \frac{2(\lambda+1)}{\left[\frac{\lambda \sqrt[n+1]{\lambda} T^{\frac{n^{2}+n-1}{n+1}}}{n !}+\frac{\lambda T^{n}}{(n+1) !}\right]},
$$

true $\forall N>\max \left\{T+|x|,\left(\frac{2}{T}\right)^{\frac{1}{\alpha}}\right\}$, large enough. 
Here using Theorem 1 we extend the domain of the application results of [1].

Remark 18. We start with the Max-product Bernstein operators ([3], p. 10)

$$
B_{N}^{(M)}(f)(x)=\frac{\bigvee_{k=0}^{N} p_{N, k}(x) f\left(\frac{k}{N}\right)}{\bigvee_{k=0}^{N} p_{N, k}(x)}, \forall N \in \mathbb{N},
$$

$p_{N, k}(x)=\left(\begin{array}{c}N \\ k\end{array}\right) x^{k}(1-x)^{N-1}, x \in[0,1], \bigvee$ stands for maximum, and $f \in C_{+}([0,1])=\left\{f:[0,1] \rightarrow \mathbb{R}_{+}\right.$is continuous $\}$.

From [1] we get

$$
B_{N}^{(M)}\left(|\cdot-x|^{m}\right)(x) \leq \frac{6}{\sqrt{N+1}}, \forall x \in[0,1], m, N \in \mathbb{N} .
$$

Denote by

$C_{+}^{n}([0,1])=\left\{f:[0,1] \rightarrow \mathbb{R}_{+}, n\right.$-times continuously differentiable $\}, n \in \mathbb{N}$.

We give

Theorem 12. Let $f \in C_{+}^{n}([0,1])$, a fixed $x \in[0,1]$ such that $f^{(i)}(x)=0$, $i=1, \ldots, n$. Then

$$
\begin{aligned}
(101) \mid B_{N}^{(M)}(f)(x) & -f(x) \mid \leq \frac{\omega_{1}\left(f^{(n)},\left(\frac{6}{\sqrt{N+1}}\right)^{\frac{1}{n+1}}\right)}{n !} \\
& \times\left[\frac{6}{\sqrt{N+1}}+\frac{1}{(n+1)}\left(\frac{6}{\sqrt{N+1}}\right)^{\frac{n}{n+1}}\right], \forall N \in \mathbb{N} .
\end{aligned}
$$

We $\operatorname{get} B_{N}^{(M)}(f)(x) \rightarrow f(x)$, as $N \rightarrow \infty$.

Proof. Use of (16) for $\delta=\left(\frac{6}{\sqrt{N+1}}\right)^{\frac{1}{n+1}}$.

We continue with

Remark 19. Here we focus on the truncated Favard-Szász-Mirakjan operators

$$
K_{N}^{(M)}(f)(x)=\frac{\bigvee_{k=0}^{N} s_{N, k}(x) f\left(\frac{k}{N}\right)}{\bigvee_{k=0}^{N} s_{N, k}(x)}, x \in[0,1]
$$

$N \in \mathbb{N}, f \in C_{+}([0,1]), s_{N, k}(x)=\frac{(N x)^{k}}{k !}$, see $[3]$, p. 11 . 
From [1] we get

$$
K_{N}^{(M)}\left(|\cdot-x|^{m}\right)(x) \leq \frac{3}{\sqrt{N}}, \quad \forall x \in[0,1], N \in \mathbb{N}, m \in \mathbb{N} .
$$

We give

Theorem 13. Let $f \in C_{+}^{n}([0,1]), x$ fixed in $[0,1]$ such that $f^{(i)}(x)=0$, $i=1, \ldots, n$. Then

$$
\begin{aligned}
& \left|K_{N}^{(M)}(f)(x)-f(x)\right| \leq \frac{\omega_{1}\left(f^{(n)},\left(\frac{3}{\sqrt{N}}\right)^{\frac{1}{n+1}}\right)}{n !} \\
& \times\left[\frac{3}{\sqrt{N}}+\frac{1}{(n+1)}\left(\frac{3}{\sqrt{N}}\right)^{\frac{n}{n+1}}\right], \quad \forall N \in \mathbb{N} .
\end{aligned}
$$

Proof. Use of (16) for $\delta=\left(\frac{3}{\sqrt{N}}\right)^{\frac{1}{n+1}}$.

We make

Remark 20. Next we study the truncated Max-product Baskakov operators (see [3], p. 11)

$$
U_{N}^{(M)}(f)(x)=\frac{\bigvee_{k=0}^{N} b_{N, k}(x) f\left(\frac{k}{N}\right)}{\bigvee_{k=0}^{N} b_{N, k}(x)},
$$

$x \in[0,1], f \in C_{+}([0,1]), N \in \mathbb{N}$, where

$$
b_{N, k}(x)=\left(\begin{array}{c}
N+k-1 \\
k
\end{array}\right) \frac{x^{k}}{(1+x)^{N+k}} .
$$

We give

Theorem 14. Let $f \in C_{+}^{n}([0,1]), x \in[0,1]$ fixed, such that $f^{(i)}(x)=0$, $i=1, \ldots, n, n \in \mathbb{N}$. Then

$$
\begin{gathered}
\text { (106) }\left|U_{N}^{(M)}(f)(x)-f(x)\right| \leq \frac{\omega_{1}\left(f^{(n)},\left(\frac{2 \sqrt{3}(\sqrt{2}+2)}{\sqrt{N+1}}\right)^{\frac{1}{n+1}}\right)}{n !} \\
\times\left[\frac{2 \sqrt{3}(\sqrt{2}+2)}{\sqrt{N+1}}+\frac{1}{(n+1)}\left(\frac{2 \sqrt{3}(\sqrt{2}+2)}{\sqrt{N+1}}\right)^{\frac{n}{n+1}}\right], \\
\forall N \in \mathbb{N}-\{1\} .
\end{gathered}
$$


Proof. Use of (16) for $\delta=\left(\frac{2 \sqrt{3}(\sqrt{2}+2)}{\sqrt{N+1}}\right)^{\frac{1}{n+1}}$, we use that (see [1])

$$
\left(U_{N}^{(M)}\left(|\cdot-x|^{m}\right)\right)(x) \leq \frac{2 \sqrt{3}(\sqrt{2}+2)}{\sqrt{N+1}}, \quad N \geq 2, N \in \mathbb{N}
$$

We make

Remark 21. Here we study Max-product Meyer-Köning and Zeller operators (see [3], p. 11) defined by

(108) $Z_{N}^{(M)}(f)(x)=\frac{\bigvee_{k=0}^{\infty} s_{N, k}(x) f\left(\frac{k}{N+k}\right)}{\bigvee_{k=0}^{\infty} s_{N, k}(x)}, \forall N \in \mathbb{N}, f \in C_{+}([0,1])$,

$s_{N, k}(x)=\left(\begin{array}{c}N+k \\ k\end{array}\right) x^{k}, x \in[0,1]$.

From [1] we get

$$
Z_{N}^{(M)}\left(|\cdot-x|^{m}\right)(x) \leq \frac{8(1+\sqrt{5})}{3} \frac{\sqrt{x}(1-x)}{\sqrt{N}}=: \rho(x),
$$

$\forall x \in[0,1], N \geq 4, \forall m \in \mathbb{N}$.

We finish with

Theorem 15. Let $f \in C_{+}^{n}([0,1]), n \in \mathbb{N}, x \in[0,1], f^{(i)}(x)=0$, $i=1, \ldots, n$. Then

$$
\begin{aligned}
\mid Z_{N}^{(M)}(f)(x) & -f(x) \mid \leq \frac{\omega_{1}\left(f^{(n)},(\rho(x))^{\frac{1}{n+1}}\right)}{n !} \\
\times & {\left[\rho(x)+\frac{(\rho(x))^{\frac{n}{n+1}}}{(n+1)}\right], \forall N \geq 4, N \in \mathbb{N} . }
\end{aligned}
$$

Proof. Use of (16) with $\delta=(\rho(x))^{\frac{1}{n+1}}$.

\section{References}

[1] Anastassiou G., Approximation by Sublinear Operators, submitted, 2017.

[2] Anastassiou G., Coroianu L., Gal S., Approximation by a nonlinear Cardaliaguet-Euvrard neural network operator of max-product kind, J. Computational Analysis 85 Applications, 12(2)(2010), 396-406. 
[3] Bede B., Coroinu L., Gal S., Approximation by Max-Product type Operators, Springer, Heidelberg, New York, 2016.

[4] FEJÉR L., Über Interpolation, Göttingen Nachrichten, (1916), 66-91.

[5] Moldovan E., Observations sur certains procédés d'interpolation généralisés, (Romanian, Russian and French summaries), Acad. Republicii Pop. Romane Bul. Stiint. Sect. Stiint. Mat. Fiz., 6, (1954), 477-482.

[6] Popoviciu T., On the proof of Weierstrass' theorem with the interpolation polynomials, Acad. Republicii Pop. Romane, "Lucrarile sesiunii generale stiintifice din 2-12 Iunie 1950". 1-4(1950), 1664-1667 (in Romanian)

George A. Anastassiou

Department of Mathematical Sciences

UNIVERSITY OF MEMPHIS

Memphis, TN 38152, U.S.A.

e-mail: ganastss@memphis.edu

Received on 12.09.2017 and, in revised form, on 16.07.2018. 